\title{
A Utility-Based Auction Cooperation Incentive Mechanism in Peer-to-Peer Networks*
}

\author{
Jiujun Cheng ${ }^{1,2}$, Yuhong $\mathrm{Li}^{2}$, Weiwei Jiao ${ }^{2}$, and Jian $\mathrm{MA}^{3}$ \\ ${ }^{1}$ College of Electronics \& Infromation Engineering, TongJi University, \\ No. 4800 Cao An Road, 201804, Shanghai, China \\ ${ }^{2}$ State Key Laboratory of Networking \& Switching Beijing University of Posts \& \\ Telecommunications, 100876, Beijing, China \\ ${ }^{3}$ NOKIA Research Center, 100013, Beijing, China \\ chengjjabupt.edu.cn
}

\begin{abstract}
In P2P network, there are a considerable proportion of free riders, which contribute nothing or little to the $\mathrm{P} 2 \mathrm{P}$ system but obtain resources from the system. In order to address this problem, we present a utility-based auction cooperation incentive mechanism in the $\mathrm{P} 2 \mathrm{P}$ network. Our approach is that before communicating with destination peer, the source peer first demands peers to auction their utilities, and then select the maximum utility as its destination peer, and gives some benefit to it, at the same time, gives some punishment to the liars. By using this mechanism, the more the peers contribute to the system, the more utility the peers can obtain, and therefore, the more benefits the peers can gain from the system. The experiment results show that the incentive mechanism assures the justice of gaining benefits for different kinds of peers in the system, and hence, controls the free riders effectively, and improves the whole performance of the P2P network.
\end{abstract}

Keywords: P2P, Free riders, BitTorrent, Auction, Incentive mechanism .

\section{Introduction}

In most $\mathrm{P} 2 \mathrm{P}$ network, the incentive mechanism is lacked for promoting the peers to contribute more resources to the system. It results in more and more peers in the P2P system do nothing for the system but gain more benefits from the system. This kind of peers is called free riders[1]. The so called free riders are those peers who themselves do not supply any resources to the system but can obtain resources from the P2P system.

The data in [2] shows that, in Napster system, about $40-60 \%$ of the peers share only $5-20 \%$ of the shared files, and $20-40 \%$ of peers share little or no files. In Gnutella system, as high as $25 \%$ of the peers do not share any files. Furthermore, about $75 \%$ of the clients share 100 files or less, whereas only $7 \%$ of the clients share

\footnotetext{
* Supported by (1) the Projects "QoS Guarantee in Hetergeneous Wireless Networks ", funded by the Chinese Ministry of Education for Returnee of Studying Abroad; (2) NOKIA-BUPT Cooperated Project (2004-2006); (3) The National Basic Research Program of China (No. 2003CB314806).
} 
more than 1000 files. The fact illustrates that in spite of claim that every peers is both a server and a client in the P2P system, a large percentage of peers rely on a small percentage of servers. Free riders can possibly obtain $20 \%$ of the maximum downloading rate in the BitTorrent [3] system.

[4] proposes a rank-based peer-selection mechanism for Peer-to-Peer media streaming systems. The mechanism provided the incentives for the cooperation through service differentiation. The free riders are given limited options in the peer selection. If a free rider gets any chance for downloading, it can only receive a few pieces of the media with low quality. [5] adopts ideas from Game Theory to study the interaction of strategic and rational peers, and proposes a differential service-based incentive scheme to improve the system's performance. [6] describe a simple Selfish Link-based Incentive(SLIC) mechanism for the unstructured P2P file sharing systems. It creates an incentive model for exchanging service better. In this model, the peers can encouraged to share more data, and give more capabilities to handle other peers' queries, as well as establish more connections to improve the P2P overlay network.

In this paper, we present a new and challengeable incentive mechanism based on utility, which is different from the mechanisms involved above. By using the mechanism, the more the peers contribute to the system, the more utility the peers can get, and consequently have more chance to win in the system and obtain more benefits. At the same time, it can promote more peers to do their best to contribute to the system, and control the free riders effectively.

The remainder of the paper is organized as follows. In section 2, the free riders of the BitTorrent system are analyzed. In section 3, a utility-based auction cooperation incentive mechanism in P2P network is presented. The performance analysis and simulation results are depicted in section 4. Finally, conclusion are made in section 5.

\section{Free Riders in BitTorrent}

The idea of optimistic unchokingin BitTorrent's choking algorithm[7,8] shows that, at all times, a BitTorrent peer has a single 'optimistic unchoke', which is unchoked regardless of the current download rate from it. According to this, free riders can get an opportunity not uploading but downloading. Therefore, "optimistic unchoking" algorithm is unfair for the modem-like peers, the reason is that though this kind of peers do their best to contribute to $\mathrm{P} 2 \mathrm{P}$ system, each peer keeps connection with the peers providing maximum uploading, thus, they obtain benefits from the system through optimistic unchoking for a period of time, and the chance for obtaining the benefits is the same as free riders. If free riders are the ADSL-like peers, the benefits they obtained is $n$ times the size of that of modem-like peers, where $n \geq 7$.

In BitTorrent system, there are three kinds of peers we discuss, they are respectively ADSL-like, modem-like, and free riders (assume that they are ADSL-like peers). According to the different number of peers and the different proportion of three kinds of peers in $\mathrm{P} 2 \mathrm{P}$ system, we discuss respectively the download process of three kinds of peers. Here, assume that the uploading velocity and downloading velocity of three kinds of peers are respectively as follows, for free riders, the former is $0 \mathrm{~KB} / \mathrm{s}$, and the latter is $64 \mathrm{~KB} / \mathrm{s}$; for modem-like peers, both of them are $7 \mathrm{~KB} / \mathrm{s}$; and for ADSL-like peers, they are both $64 \mathrm{~KB} / \mathrm{s}$. In addition, assume that the size of files downloaded by all peers in P2P system is 10MB. 
During the downloading, peers leave the system with a probability of Normal distri bution $N\left(\mu, \sigma^{2}\right)$ [9], where, $\mu$ is the positional parameter in Normal distribution, and $\sigma$ describes the degree of dispersion of data distribution in Normal distribution.

Two different kinds of experiment scenarios, including 10 (free riders)-20 (Modem)-20 (ADSL), and 80 (free riders)-100(Modem)-100(ADSL) are selected respectively to describe the download process. Where, the abscissa ( $\mathrm{X}$ axis) indicates the downloading time, the unit is second, and respectively get value of three different kinds of peers at intervals of 200 seconds. The ordinate (Y axis) denotes the total data downloaded by different types of peers, and its unit is KB.

\subsection{Experiment 1}

In this experiment, assume that the total number of peers is 50. Among them, the number of free riders is 10, and the number of ADSL-like peers and Modem-like peers are both 20. According to the idea of optimistic unchoking in BitTorrent system, the download process of three different kinds of peers can be seen from figure 1 . Here, all of the peers start to download at the same time and the initial values are zero. In figure 1 , it can be seen that before free riders have finished downloading, the total data downloaded by free riders is always several times as much as that of modem-like peers at the same moment. Obviously, it is unfair for the modem-like peers trying their best to contribute to the system.

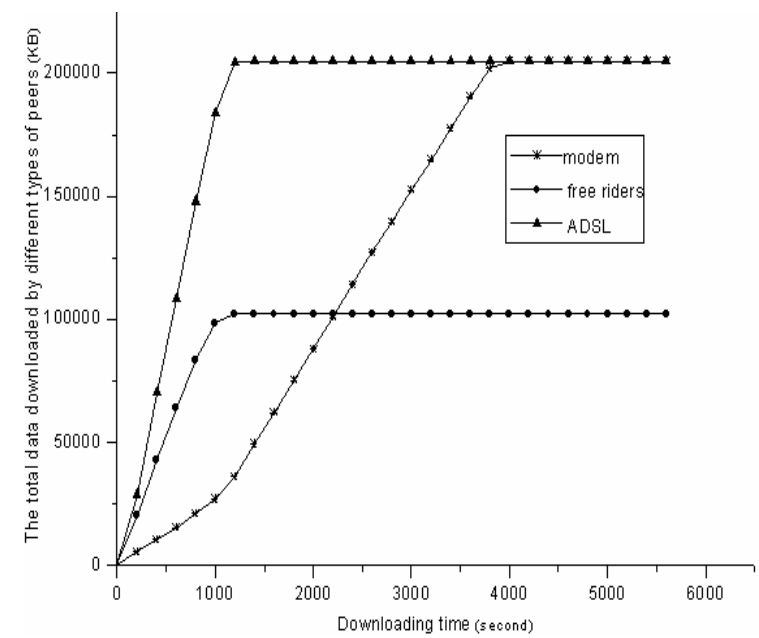

Fig. 1. The download process of three kinds of peers in the system with 50 peers

\subsection{Experiment 2}

Assume that the total number of peers is 280 . Among them, the number of free riders is 80, and the number of ADSL-like peers and Modem-like peers are both 100 . According to the idea of optimistic unchoking in BitTorrent system, the download process of different kinds of peers is shown as figure 2. The time that ADSL-like 
peers have finished downloading is 10,600s, and the total data downloaded is 1.024E6 $\mathrm{KB}$; the time needed by free riders is 10,400 s, and the total data downloaded is $819,200 \mathrm{~KB}$; the time needed by modem-like is $16,600 \mathrm{~s}$, and the total data downloaded is $990,208 \mathrm{~KB}$.

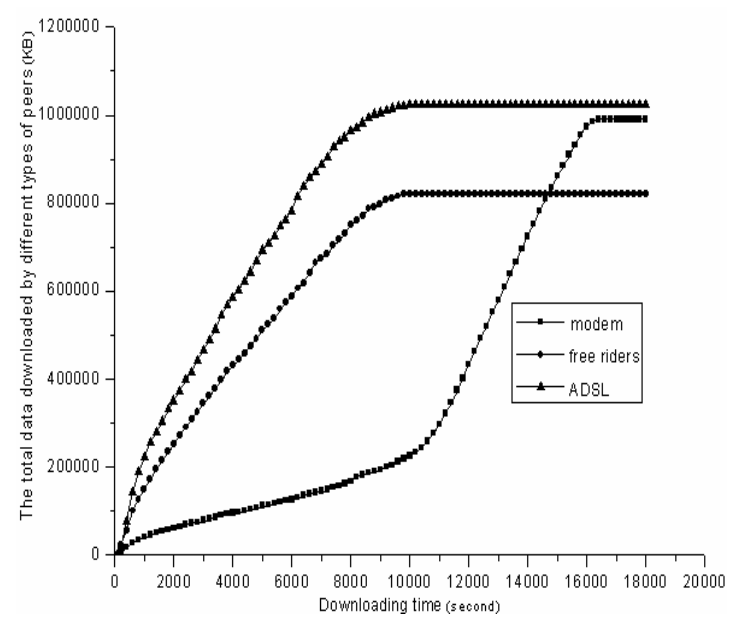

Fig. 2. The download process of three kinds of peers in the system with 280 peers

In this experiment, the ADSL-like peers and the modem-like peers would leave the system with the probability of $N\left(\mu, \sigma^{2}\right)$ Normal distribution, and this leads to the total data downloaded less than 100x10 MB when they have finished downloading. Similarly, from figure 2 , it can been that before free riders have finished downloading, the total data downloaded by free riders is several times as much as that of modem-like peers at the same moment. Obviously, it is unfair for the modem-like peers trying their best to contribute to the system.

\subsection{Experiment 3}

Assume that the number of peers is 280 in the two different P2P systems. In one system, there are 80 free riders, 100 ADSL-like peers, and 100 modem-like peers; and in the other system, there are 180 ADSL-like peers, and 100 modem-like peers. The difference between system 1 and system 2 is that system 1 has more 80 ADSL-like peers providing uploading than system 2 has.

Figure 3 describes the throughput of the two different systems. During the initial 1000 seconds, the throughput in system 2 is far more than that in system 1. Next, the throughput in the two systems is more nearer, it shows that some peers in system 2 would only provide uploading after they have finished downloading.

When the downloading time reaches 11,700 s, the throughput in system 2 closes to zero, at the moment, system 2 has finished downloading. However, the download process in system 1 has to persist 16,600s. The end of download process in system 1 


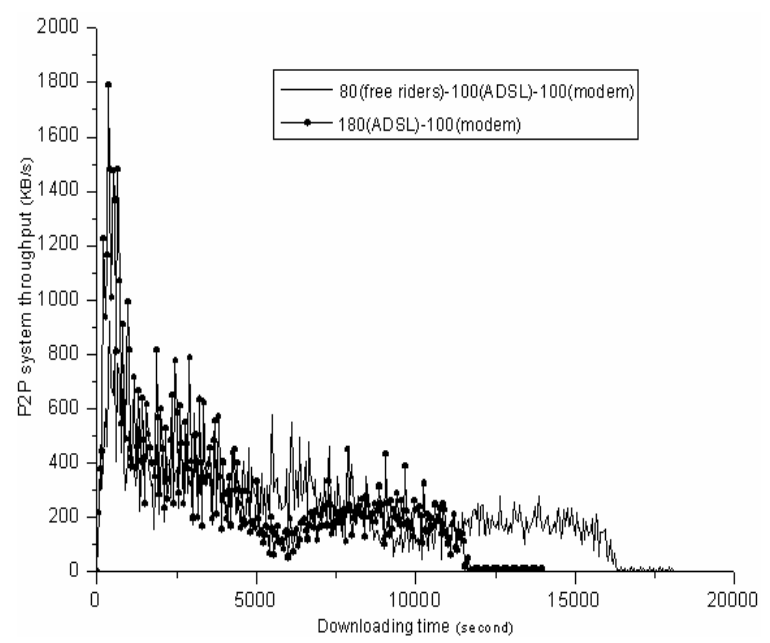

Fig. 3. The throughput of the two different systems

has later 4,900s than that in system 2, the reason is that what free riders only do is downloading but not uploading, it obviously affects the whole performance of P2P network.

\section{Utility-Based Auction Cooperation Incentive Mechanism}

In this section, we discuss a utility-based auction cooperation incentive mechanism in $\mathrm{P} 2 \mathrm{P}$ network to resolve the questions described above. The purpose of this incentive mechanism is that the more peers contribute to the system, the more utility the peers can get, and this makes them win in the competition, and consequently obtain more benefits, at the same time, those peers who are cheating during auctioning will be punished. Assume that all peers in $\mathrm{P} 2 \mathrm{P}$ system share the same file, namely, the homogenous P2P network (here, the homogenous P2P network means that all peers which share the same file compose of a P2P network).

Assume that there are $N$ peers in P2P network, they are respectively $P_{1}, P_{2}, \cdots, P_{N}$, and $B(i)$ is the total cost paid by peer $P_{i}$ which downloads different pieces from other peers, and $B(i, j)$ denotes the cost paid by peer $P_{i}$ downloaded pieces from peer $P_{j}$, if peer $P_{i}$ takes no interests in peer $P_{j}$, then $B(i, j) i s 0$, in the same way, for all peers $P_{i}, B(i, i)$ is 0 . Therefore, the total cost $B(i)$ paid by peer $P_{i}$ is given by

$$
B(i)=\sum_{j=1}^{N} B(i, j)=\sum_{j=1}^{N} d(j) D(j)
$$

Where, $d(j)$ is the cost paid by a peer for uploading or downloading a piece; $D(j)$ denotes the size of resource that peer $P_{i}$ contributes to peer $P_{j}$. 
Likewise, when a peer supplies resource to many peers in the system, the benefit that the peer obtains is assumed as $C(i)$, where, $C(i, j)$ denotes the income obtained by peer $P_{i}$ which supplies resource to peer $P_{j}$, if peer $P_{i}$ isn't interested in peer $P_{j}$, then $C(i, j)$ is 0 , in the same way, for all peers $P_{i}, C(i, i) i s 0$. Thus, the total income $C(i)$ obtained by peer $P_{i}$ is given by

$$
C(i)=\sum_{j=1}^{N} C(i, j)=\sum_{j=1}^{N} d(j) M(j)
$$

Where, $M(j)$ denotes the size of resource that peer $P_{i}$ contributes to peer $P_{j}$.

Assume that $U(i)$ is the utility of peer $P_{i}$ participating in the competition, $R(i)$ denotes the rewards of peer $P_{i}$ after it supplied resource to the system, then the utility $U(i)$ of peer $P_{i}$ is as follows:

$$
U(i)=-B(i)+C(i)+R(i)
$$

Initially, all peers' utilities $U(i)(1 \leq i \leq N)$ are zero, namely, peers not only upload nothing, but also download nothing.

In P2P network, the communication between peers is classified as two kinds, one is the direct communication between peers, such as the communication after searching through Gnutella protocol; the other is the communication between peers through a few intermediate transmitting peers, such as in Ad Hoc network. We analyze respectively the incentive mechanism between peers for the two situations involved.

\subsection{The Incentive Mechanism on the Direct Communication Between Peers}

Definition 1. In the homogenous $\mathrm{P} 2 \mathrm{P}$ network, if peer A and peer B can communicate directly with each other without any intermediate transmitting peers, then we call that peer $\mathrm{A}$ and peer $\mathrm{B}$ form a 0-related adjacent grouped pair, and mark it as $A \stackrel{0}{\sim} B$.

A source peer downloads directly data from many destination peers is the same as many source peers download directly data from a destination peer. Here, we consider only the former.

Before communicating with destination peers, the source peer firstly demands destination peers to mark their utilities when they participate in this competition, they are respectively $U(1), U(2), \cdots, U(k)$, which is used to win in the competition and finally communicates with the source peer. Initially, $U(i)(1 \leq i \leq N)$ are all zero, and the source peer decides to select the superior destination peer to communicate with it according to the uploading velocity which the destination peers provide.

In order to promote more peers to contribute to the $\mathrm{P} 2 \mathrm{P}$ system, it needs to give rewards for the peer supplying the sharing resource, and it is assumed as $R(i)$, where, $R(i)$ is the difference value between the maximum utility $U_{\max }$ and the hypomaximum utility $U_{\max }^{\prime}$, that is: 


$$
R(i)=U_{\max }-U_{\max }^{\prime}
$$

At the moment, the utility $U^{\prime}(i)$ of destination peer is given by

$$
U^{\prime}(i)=U(i)+R(i)
$$

According to the formula (5), if $U^{\prime}(i)<U(i)$, that is $R(i)<0$, it shows that the peer wins in the competition is an dishonest one. In order to win the competition, the peer deliberately bids up its utility $U(i)$, and consequently makes its utility be higher than the actual maximum utility $U_{\max }$ among the destination peers. According to (4), the actual rewards it obtained is minus, thus, the source peer won't communicate with it, and gives it corresponding punishment, namely, subtracts the utility $U(i)$ of peer from $|R(i)|$, thus, the utility $U^{\prime}(i)$ of the dishonest peer is given by

$$
U^{\prime}(i)=U(i)-|R(i)|
$$

if $U^{\prime}(i)>U(i)$, that is $R(i)>0$, it shows that the peer wins in the competition is an honest one, thus, the source peer will communicate with it, and the utility $U^{\prime}(i)$ of the destination peer can be given by

$$
U^{\prime}(i)=U(i)+C(i)+R(i)
$$

In addition, the utility $U^{\prime \prime}(i)$ of source peer is given by

$$
U^{\prime \prime}(i)=U(i)-B(i)
$$

When the source peer and the destination peers all have new utilities, they begin to enter into next competition.

\subsection{The Incentive Mechanism on the Communication Between Peers Through Intermediate Transmitting Peers}

Definition 2. In the homogenous P2P network, if the communicate between peer A and peer $\mathrm{B}$ need intermediate transmitting peers, and the relations among them are: $A \stackrel{0}{\sim} I_{1}, I_{1} \sim I_{2}, \cdots, I_{j} \sim I_{j+1}, \cdots, I_{k} \sim B$, then we call that peer A and peer B form a $k$ related adjacent grouped pair, and mark it as $A_{\sim}^{k} B$.

In wireless network, the communication between two peers maybe need one or more intermediate transmitting peers, in order to promote more peers contribute to the P2P system, the utility auction are used to select the optimal path during the router selection. We adopt the $\mathrm{P} 2 \mathrm{P}$ searching protocol to find all destination peers, and form a network topology. Here, we consider the source peer downloading from destination peers, and the router selection process starts from destination peers, the concrete algorithm describes as follows:

(1) At first, the destination peer demands its adjacent peers to mark their utilities when they participate in this competition, they are respectively $U(1), U(2), \cdots, U(k)$, 
which is used to win in the competition and finally communicates with the destination peer, and the process of competition is described in section 3.1.

Assume that the winner of this competition is $P_{j}$, and it is also used as the source peer in the next competition. Because the intermediate transmitting peers upload data, and at the same time they download data, therefore, the costs of both of them are counteracted. The utility of $P_{j}$ is $U^{\prime}(j)$ :

$$
U^{\prime}(j)=U(j)+R(j)
$$

where, $U(j)$ denotes the utility of $P_{j}$ participating in this competition, $R(j)$ is the rewards of peer $P_{j}$. Here, it also assures that the intermediate transmitting peers with the honest deed participate in the competition. The winner peer $P_{j}$ and the destination peer B are placed into the optimal router set $V$, and the set $V$ is $\left\{P_{j}, B\right\}$;

(2) The process of the peer $P_{j}$ selecting the next optimal peer is the same as (1), that is to say, peer $P_{j}$ selects the maximum utility peer as transmitting peer from its adjacent peers, and the peer will obtain some rewards as a return, and then place it to the optimal router set $V$;

(3) Repeat the process (2), and place all the peers which are up to the mustard into the optimal set $V$, until the source peer is reached;

(4) The set $V$ is the optimal path from the source peer to the destination peers.

\section{Performance Analysis and Experiment Results}

In this section, to those different scenarios described in section 2, we analyze the differences in each scenario before and after the incentive mechanism is applied. Where, we only consider the direct communication among peers. The download processes of different types of peers are shown in figure 4 and figure 5 .

In figure 4, the download processes of 50 peers (among them, 10 are free riders, 20 are modem, and 20 are ADSL) are described before and after the incentive mechanism is applied. After the incentive mechanism is applied (denoted by 0-20-20 in figure 4), the free riders have not obtained any utility during the competition, since they have not made any contribution to the system. Therefore, during the whole downloading, the data quantity received by the download processes of ADSL-like peers and modem-like peers have changed greatly.

For the ADSL-like peers, the download time decreases from 1400s to 1000 s after the utility-based incentive mechanism is adopted. And for the modem-like peers, the data quantity used the incentive mechanism is always more than that without using the incentive mechanism. The reason for such phenomena is that only ADSL-like peers and modem-like peers participate in the competition during the downloading after the incentive mechanism is applied. Because the upload rate of ADSL-like peers is $n(n \geq 7)$ times as much as that of modem-like peers, ADSL-like peers obtain 


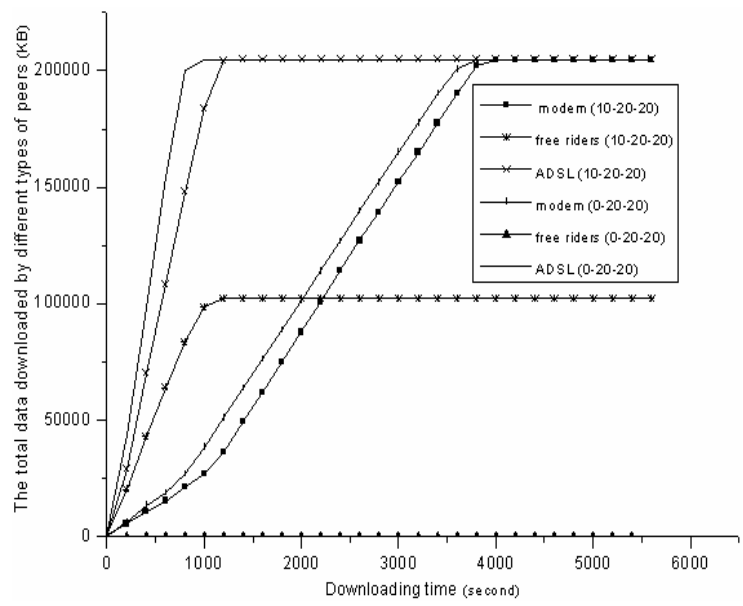

Fig. 4. The download processes of three types of peers in the system with 50 peers before and after the incentive mechanism is applied

greater utility value than that of other peers and have more chance to win in the competition. Hence, the time used for downloading is shorter.

At the beginning, only when modem-like peers do not compete with ADSL-like peers for the same data, they have the chance to win. When ADSL-like peers have finished downloading completely, only modem-like peers alone exist in P2P system. Thus, compared with before the incentive mechanism is applied, the total data downloaded by the modem-like peers has increased evidently, and during the downloading, the leaving probability of modem-like peers decreases obviously. When

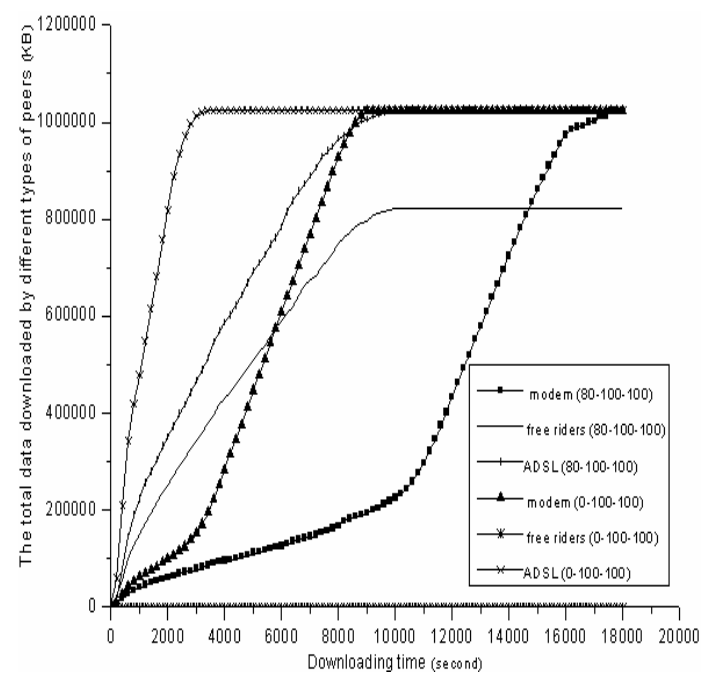

Fig. 5. The download processes of three types of peers in the system with 280 peers before and after the incentive mechanism is applied 
the system download process finished, the total data downloaded by modem-like peers is nearly up to $200 \mathrm{MB}$.

In figure 5, the download processes of 280 peers (among them, 80 are free riders, 100 are modem, and 100 are ADSL-like peers) are described before and after the incentive mechanism is applied. After the incentive mechanism is applied (denoted by 0-100-100 in figure 5), the total data downloaded by the free riders always keeps zero during the whole downloading. Before the incentive mechanism is adopted, the time that ADSL-like peers have finished downloading is $10,600 \mathrm{~s}$. However, after the incentive mechanism is applied, the time decreased to 3,400s, which is less than one third than that before. And for the modem-like peer, before the incentive mechanism is applied, the time needed to finish downloading is 16,600s and the total data downloaded is $990,208 \mathrm{~KB}$; after the incentive mechanism is applied, the time needed is $9,200 \mathrm{~s}$ and the total data downloaded is $1,024,000 \mathrm{~KB}$.

From figure 5, it can be seen that during the initial period of download, the total data downloaded by ADSL-like peers occupies a high percentage, while in the same processes, the data downloaded by modem-like peers occupies a lower percentage. When all the ADSL-like peers finish downloading, the number of the seeds in the P2P system increases accordingly. Meanwhile, only modem-like peers alone participate in the competition in the system, thus, the total data downloaded by modem-like peers rises sharply. When all the modem-like peers have finish downloading, the total data downloaded is nearly up to $100 \times 10 \mathrm{MB}$.

\section{Conclusion}

According to the idea of optimistic unchoking of BitTorrent, the free riders in the P2P system have the same chance for obtaining the benefits as other modem-like peers, it is unfair for the modem-like peers who do their best to provide maximum uploading velocity for the system. In this paper, we first discussed the two scenarios on the download process of three different kinds of peers, and the simulation results show that the free riders affect not only the downloading time but also the total data downloaded for other peers.

Based on the discussion, a utility-based auction cooperation incentive mechanism in $\mathrm{P} 2 \mathrm{P}$ network is then proposed. By using this mechanism, the more the peers contribute to the system, the more utility the peers can get, and consequently they have more chance to win in the system, and obtain more benefits. This mechanism can control the free riders effectively, and avoid the peers' vicious deed. The simulation results demonstrate that the performance of ADSL-like and modem-like peers is improved obviously after adopting the proposed incentive mechanism, which assures the justice of different kinds of peers. And this mechanism can promote more peers to do their best to contribute to the system, and act as servers, and it can improve the whole performance of $\mathrm{P} 2 \mathrm{P}$ network.

\section{References}

1. E.Adar and B. Huberman. Free riding on gnutella. First Monday, 5 (10), October 2000.

2. Stefan Saroiu, P.Krishna Gummadi, Steven D. Gribble. A Measurement Study of Peer-toPeer File Sharing Systems. Multimedia Computing and Networking 2002 (MMCN'02). 
3. Dongyu Qiu and R.Srikant. Modeling and Performance Analysis of BitTorrent-Like Peer-toPeer Networks. ACM SIGCOMM, Aug.30-Sept.3, 2004.

4. Ahsan Habib and John Chuang. Incentive Mechanism for Peer-to-Peer Media Streaming. 2004. IEEE.

5. Chiranjeeb Buragohain, Dibyakant Agrawal and Subhash Suri. A Game Theoretic Framework for Incentives in P2P System. Proceedings of the Third International Conference on Peer-to-Peer Comptuting (P2P'03). 2003. IEEE.

6. Qixiang Sun, Hector Garcia-Molina. SLIC: A Selfish Link-based Incentive Mechanism for Unstructured Peer-to-Peer Networks. Proceeding of the $24^{\text {th }}$ International Conference on Distributed Computing Systems (ICDCS'04). 2004. IEEE.

7. http://bittorrent.com/.

8. B ram Cohen, Incentives Build Robustness in BitTorrent. Bitconjurer.org/BitTorrent, May 22, 2003.

9. Weizongshu, Probability Theory \& Mathematical Statistics [C], China Higher education press, 1997. 